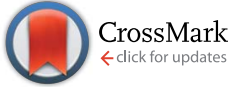

Cite this: Soft Matter, 2014, 10, 6955

Received 28th February 2014 Accepted 1st April 2014

DOI: $10.1039 / c 4 s m 00467 a$

www.rsc.org/softmatter

\section{Analysis of liquid metal foams through X-ray radioscopy and microgravity experiments $\uparrow$}

\author{
F. García-Moreno, ${ }^{\text {ab }}$ S. T. Tobin, ${ }^{c}$ M. Mukherjee, ${ }^{\text {bd }}$ C. Jiménez, ${ }^{\text {ab }}$ E. Solórzano, ${ }^{e}$ \\ G. S. Vinod Kumar, ${ }^{\text {bf }}$ S. Hutzler ${ }^{\mathrm{c}}$ and J. Banhart ${ }^{\mathrm{ab}}$
}

Complex liquid structures such as metallic foams were produced in a furnace that allowed in situ X-ray monitoring of the evolution of the structure and distribution of the liquid in the foam. The experiments were carried out during parabolic flights which provided varying levels of gravity. The evolution of the characteristic liquid fraction profiles due to gravity induced drainage was measured and analysed in terms of the foam drainage equation, obtaining viscosity and surface tension by fitting solutions of the equation to the experimental data. The surface tension of the melt in the foam was decreased up to $40 \%$. Effective viscosities of up to 139 times the viscosity of a pure bulk melt were observed. These effects could be attributed to the smaller influence of solid particles dispersed in the melt and the larger influence of the complex foam structure.

\section{Introduction}

Microgravity conditions are ideal for the study of complex liquids such as any type of liquid foams, i.e. aqueous, polymeric or metallic based, being able to produce homogeneous foams without the disturbing influence of gravity induced liquid drainage. ${ }^{1-5}$ On the other hand in situ X-ray radioscopy was demonstrated to be a powerful tool for the study of foam evolution. ${ }^{6-10}$ The combination of X-ray radioscopy and microgravity conditions offers a unique environment for fundamental studies.

In recent years, metal foams have become a subject of great interest in materials engineering. ${ }^{11}$ However, large-scale production has been hindered by the ignorance of important liquid properties such as surface tension or viscosity, an incomplete understanding of the processes by which metal foam melts stabilize and solidify, and how these processes influence the final structure of the foam. This is still a major challenge, as examining the molten system in situ verifying

anstitute of Applied Materials, Helmholtz Centre Berlin for Materials and Energy, Hahn-Meitner-Platz 1, 14109 Berlin, Germany. E-mail: garcia-moreno@ helmholtz-berlin.de

${ }^{b}$ Institute of Materials Science and Technology, Technical University Berlin, Hardenbergstrasse 36, 10623 Berlin, Germany

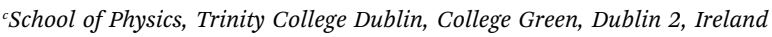

${ }^{d}$ Department of Metallurgical and Materials Engineering, Indian Institute of Technology Madras, Chennai 600 036, India

${ }^{e}$ CellMat Laboratory, Faculty of Sciences, University of Valladolid, Prado de Belén 7, 47011 Valladolid, Spain

${ }^{f}$ SRM Research Institute, SRM University, Kattankulathur, 603203, India

$\dagger$ Electronic supplementary information (ESI) available. See DOI: 10.1039/c4sm00467a proposed mechanisms requires sophisticated experimental setups such as X-ray radioscopy and microgravity conditions.

Two main mechanisms of film and foam stability have been proposed in the literature. Some authors postulate that high bulk viscosity is essential, ${ }^{12}$ while others consider the influence of solid particles to be directly responsible. ${ }^{13-16}$ These particles are theorized to be partially wetted by the liquid and therefore accumulate at the liquid-gas interfaces and/or build a kind of 'network' of interconnected particles, stabilizing the system. Unfortunately, the possible mechanisms are difficult to isolate from each other, as the presence of solid particles in the melt induces a higher viscosity. ${ }^{17}$ Detailed analyses of metal foams in the liquid (molten) state are yet to be carried out. The existing results are based on the melt properties, and microstructure analysis of solidified samples, models, or simulations.

For simulations and modelling, melt parameters such as the liquid viscosity and surface tension are required. The standard approach has been to assume that the parameters taken from the bulk liquid matrix material are valid for the metal foam. However, the contribution of the cellular structure has to be also considered. Standard measurement techniques for viscosity and surface tension require the destruction of the foam structure, and are therefore not generally useful.

It is known that the presence of impurities, especially the presence of oxygen, reduces the surface tension of an aluminium melt. ${ }^{18,19}$ Hence the literature data on surface tension of pure aluminium are not very useful when foams are concerned. They are highly impure because of the presence of oxygen and several other impurity atoms such as silicon, copper, magnesium etc. In the case of addition of $\mathrm{TiH}_{2}$ as a blowing agent, these particles also act as another impurity. Similarly, the viscosity of melts changes with the shear rate. But 
there is no knowledge of the shear rate while melt flow through the foam structure is considered.

We estimate the surface tension and viscosity of the melt of liquid metal foams extracting values from experimental data of the liquid fraction profiles, solving the foam drainage equation, ${ }^{20}$ which describes the flow of liquid in a foam, based on radioscopy analysis by varying gravity conditions realized through microgravity experiments. Parabolic flights offer a unique possibility of performing experiments under varying gravity conditions, allowing the creation of a foam with a homogeneous liquid fraction distribution under microgravity, which is a well-defined initial state for our analysis.

\section{Experimental methods}

\section{Materials}

A foamable precursor material with the composition AlSi6Cu4 (in wt $\%$ ) $+0.6 \mathrm{wt} \%$ of $\mathrm{TiH}_{2}$ was prepared by mixing the elemental powders with the blowing agent $\mathrm{TiH}_{2}$, compacting them using cold isostatic compression and finally casting them using the thixocasting method. ${ }^{21}$ Also AlSi11 +0.5 wt $\%$ of $\mathrm{TiH}_{2}$ precursors were prepared by mixing the elemental powders but compacting them at $400{ }^{\circ} \mathrm{C}$ and $300 \mathrm{MPa}$ uni-axially following a standard powder metallurgical (PM) way. ${ }^{22}$ Further precursors of AlSi9 + $0.6 \mathrm{wt} \%$ of $\mathrm{TiH}_{2}$ with 5, 10 and $20 \mathrm{vol} \%$ SiC particles were prepared using the so-called 'Formgrip' method. ${ }^{23}$ It consists in first dispersing the blowing agent in the melt and then solidifying the composite quickly. In a second step the solidified precursor will be heated up and foamed as in the standard powder metallurgical route. The samples prepared were approximately $20 \times 10 \times 4 \mathrm{~mm}^{3}$ in size to fit into the foaming crucible.

\section{$\mathrm{X}$-ray radioscopy and furnace}

Fig. 1 shows the special X-ray transparent furnace used for microgravity experiments. It was developed and manufactured in cooperation with the Swedish Space Corporation. ${ }^{5}$ It can reach temperatures of up to $700{ }^{\circ} \mathrm{C}$ with high temperature homogeneity $( \pm 1 \mathrm{~K})$. The precursor samples were foamed to a maximum size of $20 \times 10 \times 20 \mathrm{~mm}^{3}$, with a relative density of 0.2. AlSi6Cu4 and Alsi11 samples were foamed at $650{ }^{\circ} \mathrm{C}$, and AlSi9 samples containing SiC particles at $700{ }^{\circ} \mathrm{C}$.

A micro-focus X-ray source ( $5 \mu \mathrm{m}$ diameter spot size) and a flat panel detector $(50 \mu \mathrm{m}$ pixel size, $2240 \times 2368$ pixel), both from Hamamatsu, Japan, allowed X-ray radioscopy of the foaming process in situ. ${ }^{6}$ Applying a 4-fold magnification, each detector pixel contained image information from a sample area

A

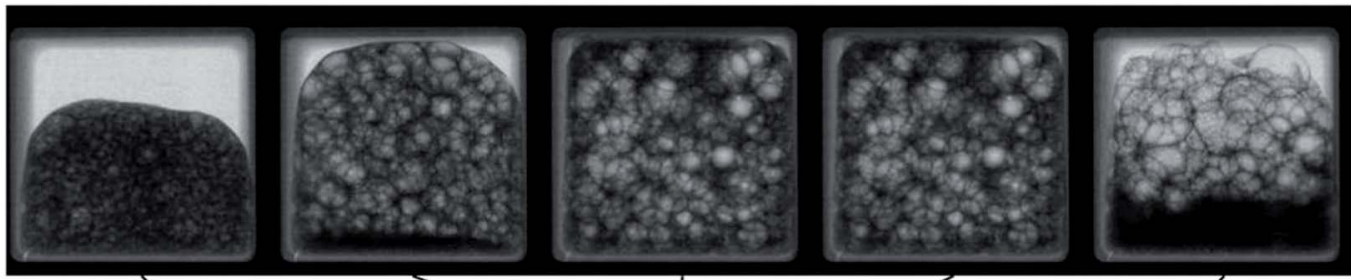

B

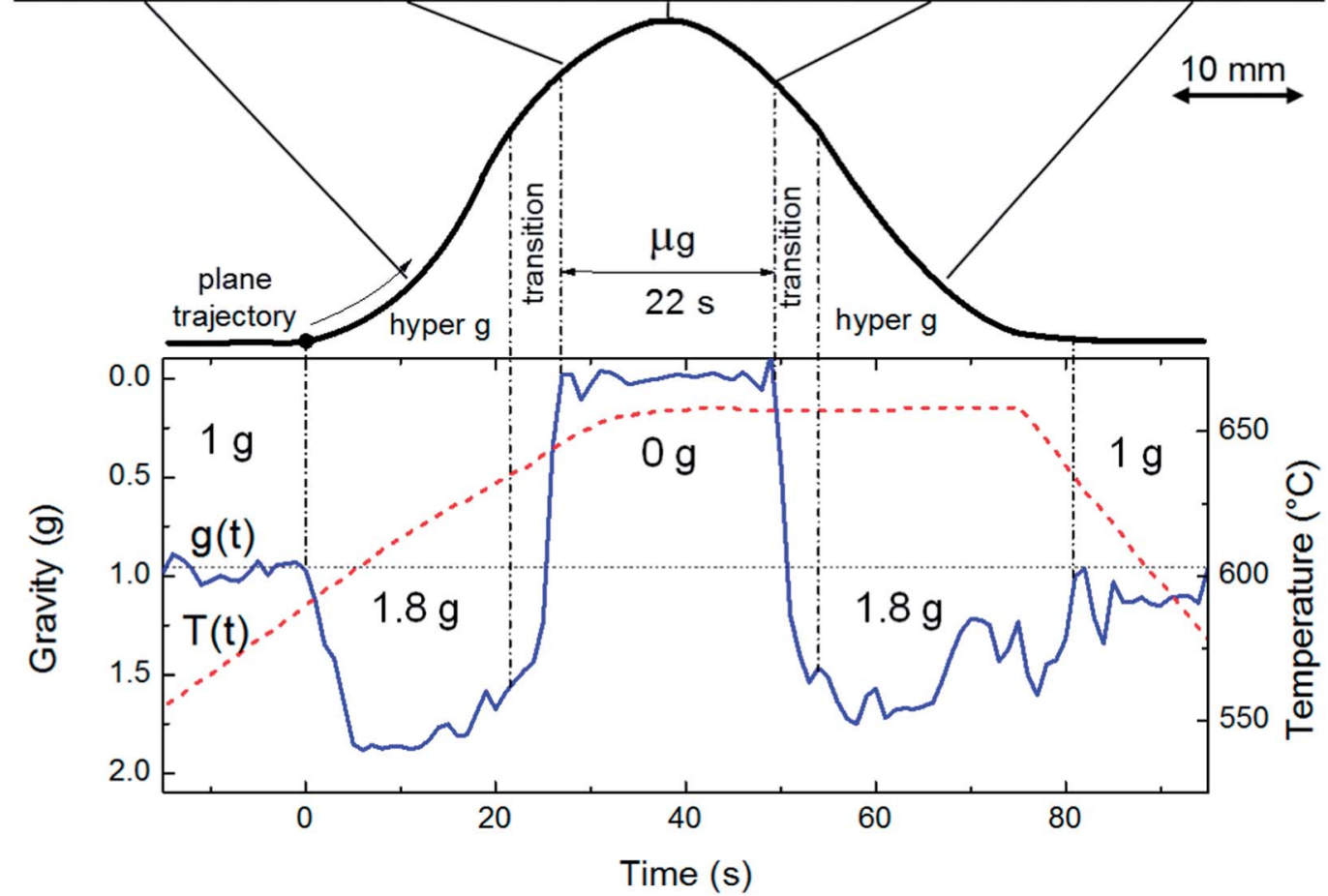

Fig. 1 Foaming process of a thixocast AlSi6Cu4 precursor material. (A) Radioscopic images are shown for different experimental stages. (B) Plane parabolic trajectory. (C) Temperature $T(t)$ (dot line) and gravity $g(t)$ (bulk line) profiles during the parabolas. 
of $12.5 \times 12.5 \mu^{2}$. The acquisition speed was $1 \mathrm{fps}$ for full spatial resolution, and max. 8 fps using $4 \times 4$ binning of the images. With the same setup and additional rotation of the foams followed by $3 \mathrm{D}$ reconstruction of the data of X-ray tomographies of the solidified foams were obtained. Further analysis allowed us to calculate a mean thickness of the walls.

\section{Microgravity experiments}

During the $46^{\text {th }}$ and $51^{\text {st }}$ parabolic flight campaigns of the European Space Agency (ESA) samples were heated from room temperature to the foaming temperature of 650 or $700{ }^{\circ} \mathrm{C}$ (depending on the alloy) over a total period of time of almost 200 $\mathrm{s}$, and foamed during the $1 g$ and $1.8 g$ phases $\left(1 g \approx 9.8 \mathrm{~m} \mathrm{~s}^{-2}\right.$ is the acceleration due to gravity) prior to a microgravity phase $(0 g)$ as shown in Fig. 1. The heating regime was synchronized so that foams almost filled the crucible during the first $1.8 g$ phase to avoid foam ageing and coarsening due to long holding times. During the subsequent microgravity periods (lasting 20-22 s) the liquid fraction became approximately homogeneous across the sample due to foam imbibition, i.e. liquid re-entering the foam. ${ }^{5}$ The aim of our experiments was to determine how drainage progresses starting from such a uniform state, as gravity varies from $0 g$ to $1.8 g$ (see Fig. 2). The furnace temperature, the gravity values and the radioscopy sequence were recorded during the whole experiments at rates of 1-8 frames per second (Fig. 1).

\section{Liquid fraction analysis}

The radioscopic images were analysed with the software package 'Axim' ${ }^{6}$ to obtain the variation of the liquid fraction in the samples. Assuming that the density of a solid foam corresponds to the liquid fraction of the liquid foam, the analysis provides 2D liquid fraction distributions of the evolving foam $(\Phi(x, z))$ by applying Beer-Lambert's attenuation law to the intensity $I(x, z)$ obtained from the radiographs:

$$
I(x, z)=I_{0} \mathrm{e}^{-\mu \Phi(x, z) d}
$$

where $\mu$ is the mass-specific absorption coefficient of the foam and $I_{0}$ the initial beam intensity. $x$ and $z$ denote horizontal and vertical coordinates, both perpendicular to the beam, and $d$ is the thickness of the foam layer. Strictly speaking this law is in general only applicable for monochromatic X-rays due to the wavelengthdependence of the absorption coefficients of different materials. But it is also applicable in good approximation for polychromatic $\mathrm{X}$-rays and almost only one single material of the base alloy, aluminium in this case, as the wavelength-dependence is same. For a constant foam depth $d$ in the beam direction the time dependent liquid fraction $\Phi$ of the foam can be calculated:

$$
\Phi(x, z, t)=\frac{\ln \left(I(x, z, t) / I_{0}\right)}{\ln \left(I_{\text {liquid }} / I_{0}\right)}
$$

where $I_{\text {liq }}$ is the transmitted intensity after attenuation of the beam by the liquid metal of thickness $d$. The key external factor affecting drainage is gravity, which acts in the (vertical) $z$ direction only. We can then average all pixel values in the horizontal $x$-direction to arrive at an integrated liquid fraction
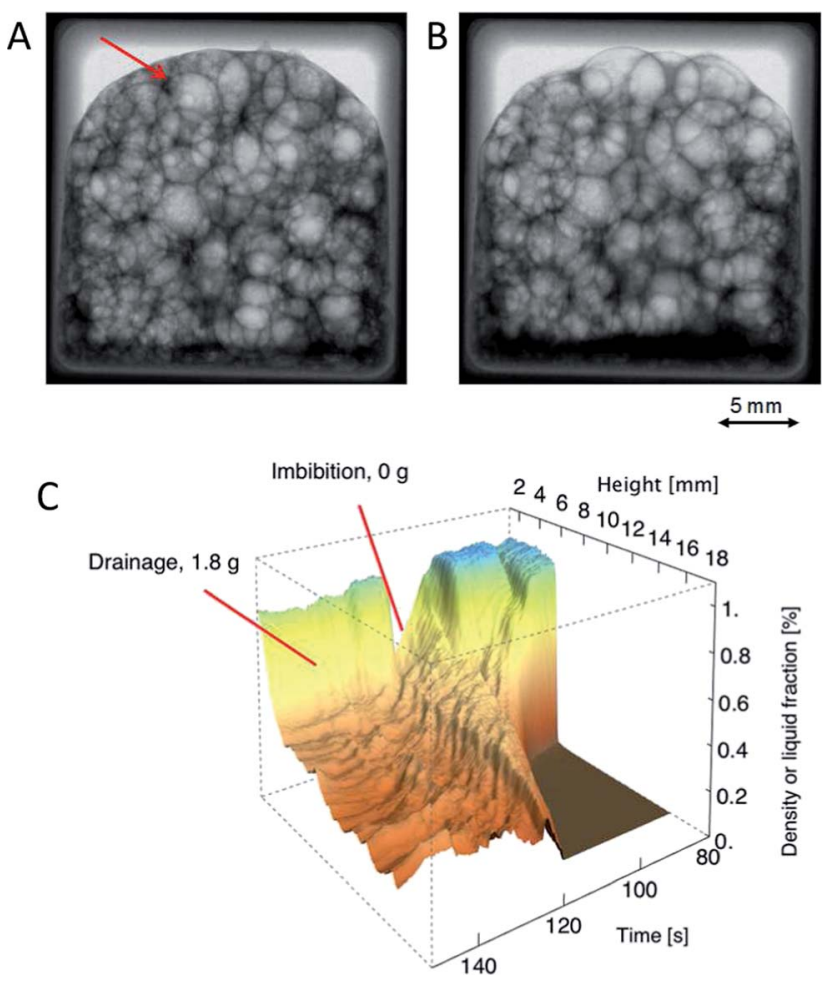

Fig. 2 X-ray radiographs of a liquid foam made of thixocast AlSi6Cu4 $+0.6 \mathrm{wt} \% \mathrm{TiH}_{2}$ precursor during the transition from (A) $\mathrm{Og}$ to (B) $1.8 \mathrm{~g}$. The elapsed time between images is $1 \mathrm{~s}$. The arrow indicates a ruptured bubble. (C) Experimentally obtained liquid fraction profiles of an AlSi6Cu4 foam during foaming. We can see the precursor melting and foaming until filling all the available space ( $80 \mathrm{~s}$ to $110 \mathrm{~s}$ ), followed by a homogenisation under microgravity (as indicated, $110 \mathrm{~s}$ to $120 \mathrm{~s}$ ). The foam then undergoes drainage as the system experiences hypergravity (120 s onwards), leading to a sharp gradient in liquid fraction.

profile which is a function of $z$ and time $t$ only (the density values in the $y$-direction are already averaged by the beam traversing the sample). This averaging process gives smoother, more representative, profiles, and can be calculated with:

$$
\Phi(z, t)=\frac{\ln \left(\frac{1}{n} \sum_{i=0}^{n} I\left(x_{i}, z, t\right) / I_{0}\right)}{\ln \left(I_{\mathrm{liq}} / I_{0}\right)}
$$

We conducted the fits of the liquid fraction profiles using the MIGRAD minimizer from the CERN MINUIT software. ${ }^{24}$ This software implements a variable-metric method with inexact line search, a stable metric updating scheme, and checks for positive-definiteness. By conducting multiple fits, each with a different starting point and evolution length, we can arrive at a more representative value for each parameter, and provide some measure of the error using the standard deviations.

\section{Results and discussion}

\section{$\mathrm{X}$-ray radioscopy}

A series of X-ray radioscopic images, with the corresponding gravity levels, were recorded during a parabola allowing for a 
qualitative observation of the metal foaming process (see Fig. 1). These images cover the changes in the foam structure during a whole parabola, i.e. $1 g \rightarrow 1.8 g \rightarrow 0 g \rightarrow 1.8 g$ $\rightarrow 1 g$. We can observe a darker region at the bottom of the samples, corresponding to pronounced gravity-induced drainage near the completion of the foam expansion of a thixocast AlSi6Cu4 foam at $650{ }^{\circ} \mathrm{C}$ during the first $1.8 g$ phase (Fig. 1). Transition to microgravity induced a homogeneous liquid fraction distribution all over the foam in a few seconds, followed by a strong liquid drainage to the bottom of the foam at the transition from 0 to $1.8 \mathrm{~g}$. The radioscopic images of this transition were used as the input for the quantitative analysis.

Fig. 2 shows two X-ray radiographs extracted from a radioscopy series of the expanded AlSi6Cu4 foam obtained in situ during the transition from 0 to $1.8 \mathrm{~g}$. Large changes in the foam liquid distribution are apparent even though the images are separated by just 1 second, emphasising the rapidity of liquid flow due to drainage. As the upper part of the foam dries out, melt is collected at the bottom, leading to a vertical liquid fraction gradient. Despite the large liquid rearrangement, most of the bubbles can be still identified. Coalescence of bubbles was limited in this time period, with just a few events observed (one example is indicated by an arrow).

In Fig. 3a-c we show drainage in AlSi9 foams with 5, 10 and 20 vol\% of SiC particles (at $700{ }^{\circ} \mathrm{C}$, after the 0 to $1.8 \mathrm{~g}$ transition). Here the liquid flow is reduced compared to the AlSi6Cu4 foam where no particle is present, and we can observe that the larger
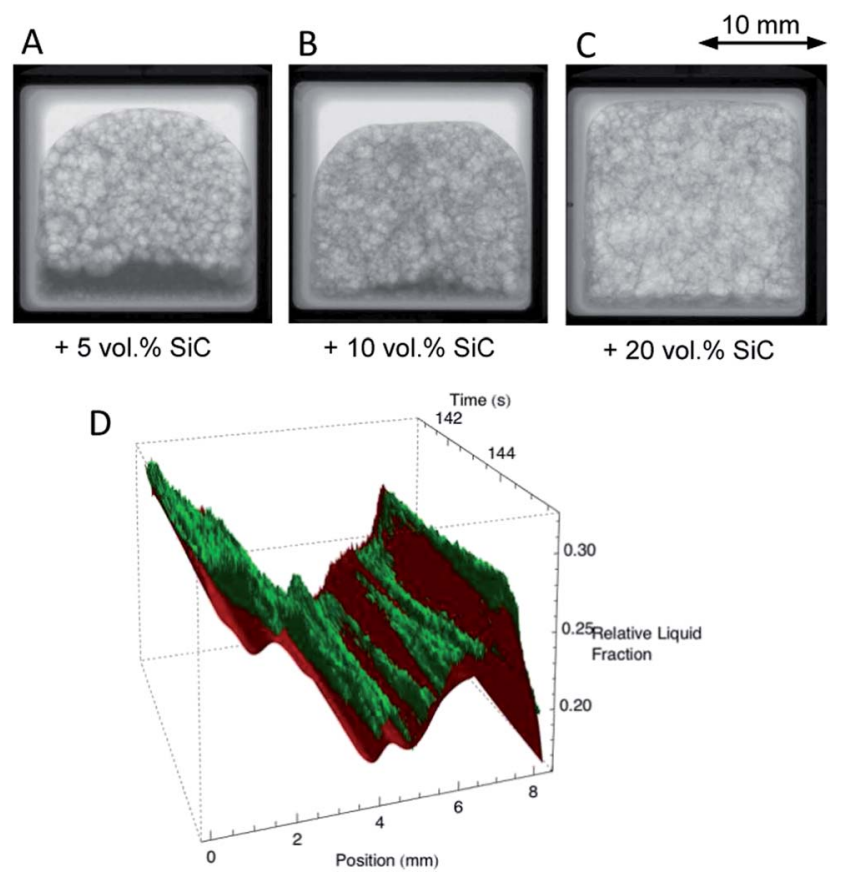

Fig. 3 X-ray radiographs of AlSi9 foams at $650{ }^{\circ} \mathrm{C}$ just after the transition from 0 to $1.8 \mathrm{~g}$. Samples containing (A) 5, (B) 10 and (C) 20 vol\% of $\mathrm{SiC}$ particles. The increased viscosity of AISi9 with increasing amount of $\mathrm{SiC}$ is apparent from the reduced liquid accumulation at the bottom of the sample. (D) An example of multiple data fit for an AlSi9 + 5 vol\% $\mathrm{SiC}$ liquid foam. Both experimental (green) and simulated (red) liquid fraction curves start from the same base liquid profile (starting at $141 \mathrm{~s}$ ). the particle content of the bulk, the less amount of melt is accumulated at the bottom part.

\section{Gravity induced drainage analysis}

The quantity and velocity of gravity-induced drainage observed for different types of foams vary depending on alloy composition, processing method and the content of ceramic particles in the precursor (see Fig. 3). At one extreme, the AlSi6Cu4 foam produced from thixocasting powder compacts shows the largest drainage effect. Such systems are known to have a low degree of stability, probably by a fragmentation of the oxide network during casting. Stability of such foams can be improved by adding particles. ${ }^{25}$ AlSi11 foams produced by standard PM processing (powder pressing only) are stabilised to a larger extent by the oxides contained in the metal powders ${ }^{\mathbf{1 3}}$ and accordingly exhibit a lower but still notable drainage effect. AlSi9 foams containing 5-20 vol\% SiC particles show a further reduced drainage with a clear dependence on the particle content.

Although drainage occurs already during foaming at $1.8 \mathrm{~g}$ (this is especially visible in Fig. 1 and 2 c for AlSi6Cu4 foam) a uniform liquid distribution is achieved during the ensuing $0 g$ stage due to imbibition of liquid into the foam under the influence of capillary forces that become dominant in the absence of gravity (see Fig. 1 and 2c). In this way, it is ensured that all the experiments start from a similar uniform liquid distribution, which is a requested starting point for drainage experiments.

In our experiments, analysis is performed within a period of just a few seconds after the transition from $0 \mathrm{~g}$ to $1.8 \mathrm{~g}$, and therefore the effect of bubble coarsening caused by gas diffusion can be neglected (as can also be observed in the X-ray image sequences, Fig. 2a and b). On the other hand, some film ruptures cannot be avoided as the X-ray images show. If we consider film rupture rates of 0.6 and $1.3 \mathrm{~s}^{-1} \mathrm{~cm}^{-3}$ for temperatures of $650{ }^{\circ} \mathrm{C}$ and $700{ }^{\circ} \mathrm{C}$, respectively (as previously found for similar alloys), ${ }^{9}$ then about $2.5-5$ ruptures per second should be observed in our samples (of about $4 \mathrm{~cm}^{3}$ ). The rupture rate was found to be hardly affected by gravity changes even for a foam such as AlSi6Cu4, which shows strong drainage (see Fig. 2b), where almost all bubbles can still be identified after a transition. From these observations we can conclude that application of the foam drainage equation is justified.

\section{The foam drainage equation}

Drainage is one of the major processes influencing foam formation and evolution. As drainage is primarily driven by gravity, the varying gravity environment of parabolic flights offers the possibility to examine foams without, with increased (gravity higher than standard) or decreased (gravity less than standard) drainage.

The foam drainage equation ${ }^{20}$ describes the flow of liquid through the Plateau border network of a foam as a function of time $t$ and vertical coordinate $z$, and a solution of the equation describes how the foam evolves in time and space without considering other disturbing processes such as coarsening or 
coalescence. The partial differential equation may then be written as

$$
\frac{\partial \Phi}{\partial t}+\frac{1}{\eta^{*}} \frac{\partial}{\partial z}\left(\rho_{\mathrm{liq}} g \frac{V_{\mathrm{b}}^{\frac{2}{3}}}{5.35} \Phi^{2}-\frac{C \gamma}{2} \sqrt{\frac{V_{\mathrm{b}^{\frac{2}{3}}}}{5.35}} \Phi \frac{\partial \Phi}{\partial z}\right)=0
$$

where $\gamma$ is the surface tension, $\rho_{\text {liq }}$ is the density of the liquid phase (which is already known) and $g$ is the acceleration due to gravity. The effective viscosity of the foam $\eta^{*}$ is proportional to the bulk liquid viscosity $\eta_{\text {liq }}$, with the coefficient of proportionality dependent on the type of flow and shape of the Plateau borders. Assuming Poiseuille-type dissipation and random orientation of the Plateau borders it corresponds to

$$
\eta^{*}=3 \times 50 \times \eta_{\text {liq }}
$$

The factor 3 arises from the 3D nature of the Plateau border network and 50 from the channel geometry. $C$ is a geometrical constant relating to the cross-sectional shape of the Plateau borders, and is given by

$$
C=\sqrt{\sqrt{3}-\frac{\pi}{2}}
$$

Finally, $V_{\mathrm{b}}$ is the average bubble volume. We approximate the foam as a collection of Kelvin cells. To estimate $V_{\mathrm{b}}$ we then note that a Kelvin cell with edge length $L$ has volume $8 \sqrt{\pi} L^{3} .5 .35$ is a geometric prefactor, which is correct for Kelvin foam ${ }^{26,27}$ and approximately accurate for other foam types.

We take multiple measurements of edge lengths from each image, and analyse images from different times during the experiment to minimize the influence of coarsening. Numerically solving this equation results in a description of how the liquid profile $\Phi(z, t)$ evolves with time from a given starting profile. This provides an opportunity to use the experimentally measured liquid profiles, coupled with numerical solutions, to determine the key parameters of the liquid metal foam. Our approach is similar to that used by Brunke et al. ${ }^{28}$

\section{Numerical analysis}

The experimental data consist of a series of liquid density profiles for each experiment. The data consist of approximately 25 seconds of usable profiles, captured at a rate of $4-8$ per second. As the frame-rate is low, there can be large variations between profiles. Due to the limited number of bubbles making up the foam melt, any bubble rupture has a large effect on the liquid profiles due to the ensuing rearrangements.

Instead of using all of these data to determine a single set of values for the parameters of the system, we split the data into multiple subsets. Each of these subsets is defined by the start time and duration, with subsets allowed to overlap (i.e., one subset may start at $t=0 \mathrm{~s}$ and run for 5 seconds, while another starts at $t=2 \mathrm{~s}$ ). This decision was taken to mitigate the effect of noise in the experimental profiles: even if one subset contains spurious data due to a bursting bubble (for example) we can still determine useful values for surface tension and viscosity from other subsets.
The starting profile from each subset is set as the 'seed' profile for our simulations and we choose seed values for the surface tension and viscosity. From these starting conditions, the foam drainage equation can be numerically integrated to generate a prediction of how that foam will evolve. We use the experimentally measured gravity values in order to take into account the variation of gravity during the experiment. The value $g(t)$ is set at every time step during the evaluation, using a precise measurement if available and interpolating otherwise.

We then fit the integrated numerical solution to experimental data starting from the same 'seed' profile. The fit is then used to update our initial guesses for the surface tension and viscosity. The process is repeated until we are satisfied that a best-fit has been achieved. Note that this approach requires many such numerical solutions, as each fitting iteration changes the initial conditions of the integration.

Numerical integration was carried out using an explicit finite difference method with forward differences. This approach is computationally efficient to solve, which is important as our algorithm requires many such solutions. Previous work found that this method is sufficiently stable for our use. ${ }^{29}$ Space and time steps were chosen to balance speed with numerical accuracy.

We compare the simulation to experiments on a grid defined by $\Delta t$, the interval at which experimental profiles were captured (time dimension) and by $\Delta z$, the vertical resolution of the detector. This comparison (over multiple experimental profiles, depending on the duration of the simulated evolution) provides the basis for using a fitting algorithm to choose the best pair of $\gamma, \eta$ values for the experimental system.

\section{Viscosity and surface tension}

We calculate surface tension and viscosity of the melt in the foam structure by treating them as fit parameters in a fit between numerical calculations and experimental data. By averaging the results over many fitting runs, we extract values for different experimental systems. The obtained results for foams of AlSi6Cu4, AlSi11 and AlSi9 + 5, 10 and $20 \mathrm{vol} \% \mathrm{SiC}$ are presented in Table 1, together with bulk values extracted from the literature for comparison. ${ }^{30-33,35}$ Note that the errors of the analysed foam properties shown are the standard deviations of the results taken over many fitting runs.

The viscosity of a AlSi6Cu4 foam was found to be around 25 and 30 times higher than those of bulk cast Al and AlSi7 respectively. For the AlSi11 foam this viscosity increase was even 40 times compared to the corresponding bulk alloy. An even higher viscosity was measured for AlSi $9+\mathrm{SiC}$ foams, with values around 65 to 120 times higher than that for $\mathrm{Al}$ and around 75 to 140 times than that for the bulk AlSi9 alloy, depending on the particle content. There is also a trend of increasing viscosity with increasing SiC concentration, almost doubling it from 5 to 20 vol\% SiC.

The bulk viscosity of a pure Al melt decreases slightly upon alloying with $\mathrm{Si}^{33}$ The viscosity also decreases with increasing temperature. For the latter effect, there are several explanations. ${ }^{34,37-39}$ For liquid metals, the Arrhenius-Andrade equation 
Table 1 Top part: surface tension and viscosity for AlSi6Cu4, AlSi11 and AlSi9 + 5, 10 and 20 vol\% SiC foams obtained by fitting numerical solutions of the foam drainage equation to experimental liquid density profiles. Bottom part: surface tension and viscosity for bulk Al, AlSi7, AlSi9 and AlSi11 alloys as taken from the literature $28,30-33,35,36$

\begin{tabular}{llll}
\hline Alloy & Condition & $T\left({ }^{\circ} \mathrm{C}\right)$ & $\sigma\left(\mathrm{N} \mathrm{m}^{-1}\right)$ \\
\hline AlSi6Cu4 0.5 wt\% oxides & Foam PM thixocast & 650 & $0.78 \pm 0.02$ \\
AlSi11 0.5 wt\% oxides & PM, foam & 650 & $0.65 \pm 0.02$ \\
AlSi9 + 5 vol\% SiC & Bulk, foam & 700 & $0.53 \pm 0.05$ \\
AlSi9 + 10 vol\% SiC & Bulk, foam & 700 & $0.57 \pm 0.04$ \\
AlSi9 + 20 vol\% SiC & Bulk, foam & 700 & $0.61 \pm 0.04$ \\
Al & Bulk, cast & 660 & $-37 \pm 3$ \\
Al & Bulk, cast & 700 & 0.869 (ref. 31) \\
AlSi7 & Bulk, cast & 700 & 0.857 (ref. 31) \\
AlSi7 0.5 wt\% oxides & PM precursor & 700 & 0.854 (ref. 31) \\
AlSi9 & Bulk, cast & 700 & - \\
AlSi11 & Bulk, cast & 650 & 0.849 (ref. 31) \\
AlSi11 & Bulk, cast & 700 & 1.38 (ref. 30) \\
\hline
\end{tabular}

is considered to be the most adequate description, where the temperature dependence can be expressed by: $:^{\mathbf{3 9 0}}$

$$
\eta_{\text {liq }}=A / V^{1 / 3} \exp \left(B V^{-1} T^{-1}\right)
$$

In eqn (7) $V$ is atomic volume; $A$ and $B$ are two temperature invariant constants. Thus, according to eqn (7) for a temperature difference of $50 \mathrm{~K}\left(\right.$ e.g. between $650{ }^{\circ} \mathrm{C}$ and $\left.700{ }^{\circ} \mathrm{C}\right)$ the variations in viscosity are in the range of $\approx 10 \%$. These small variations allow for a comparison of data at slightly different temperatures such as the ones listed in Table 1. Obviously, temperature variations in the mentioned range cannot be responsible for the large variations observed in foams viscosity.

The viscosity found for the AlSi11 foam is 25 times that of the precursor. In an ex situ experiment using an AlSi7 foam Brunke et al. found a viscosity 10 times higher than that of the corresponding AlSi7 PM precursor. ${ }^{28}$ They measured a viscosity of the precursor 1.4 times higher than that of the corresponding molten alloy and 1.6 times higher than values given in the literature. ${ }^{33}$ This can be explained by the presence of $0.3-$ $0.7 \mathrm{wt} \%$ resident oxides in $\mathrm{Al}$ powders that are also contained in the precursors. These oxides act as solid, stabilizing particles. ${ }^{13,41,42}$

Melts with solid particles (either the solid phase in the semisolid condition or added particles) are so-called slurries and known to increase viscosity in comparison to the corresponding pure alloys. In the case of composite alloys including high percentages of SiC Flemings found that the viscosity of a AlSi6.5 alloy was increased to 2 or 3.3 times the original value by adding 10 or 20 vol\% of SiC particles, respectively. ${ }^{36,37}$ (As the absolute values of the pure alloys quoted by Flemings do not match with other newer sources they are not included in Table 1.) This can explain the stronger drainage effect found for AlSi6Cu4 and AlSi11 foams compared to foams based on AlSi9 + SiC particles. However, this increase in viscosity still cannot justify the larger increase in viscosity found in this work from the microgravity experiments on foams. The reason for the larger deviation must be related to the structural factor since the foam is based on a multitude of thin films connected to each other by struts and nodes. Therefore, we can reconsider the definition of effective viscosity for the liquid metal in the foam $\eta_{\text {foam }}^{*}$ with the form

$$
\eta_{\text {foam }}^{*}=P S \eta_{\text {liq }}
$$

Here, $P$ is a factor related to the presence of solid particles in the melt and $S$ a structural component related to the foam structure, with $P=1$ for no particles and $S=1$ for a bulk melt. The parameters determined experimentally are summarized in Table 2. For the foamed structure we find $S \gg 1$ in all cases, with values in the range $25-55$ in the analysed foams. The obtained structural coefficients, $S$, are in excellent anticorrelation (inversely proportional) with the foam average wall thickness $d_{\mathrm{w}}$ calculated via 3D tomography. These experimental thickness values further validate the foam structure coefficients obtained.

The background of this effect is the increased internal friction in the liquid caused by the reduced thickness of the foam structure (walls and Plateaus), an additional tortuosity of the liquid in the foam structure and the interactions of the floating particles with the metal-gas interfaces. Even some of the particles adhere to the film surfaces where they also significantly contribute to metal film stabilisation. This is visualised in Fig. 4 and demonstrated experimentally. ${ }^{\mathbf{1 4}}$

The surface tensions of the AlSi6Cu4 and Alsi11 foams at $650{ }^{\circ} \mathrm{C}$ are up to $25 \%$ lower than those for bulk Al, AlSi7 and AlSi9 at $700{ }^{\circ} \mathrm{C}$ found in the literature. ${ }^{31}$ The surface tensions of

Table 2 Structure factors $S$ (increase of viscosity caused by foam structure) and particle factors $P$ (increase of viscosity by particle addition) for different alloys calculated by combining our experimental values for the melt in foams and literature values ${ }^{28,36}$ for the viscosity of bulk alloy melts with and without particle addition

\begin{tabular}{lllllr}
\hline Alloy & $T\left({ }^{\circ} \mathrm{C}\right)$ & $d_{\mathrm{w}}(\mu \mathrm{m})$ & $P$ & $S$ & $P \cdot S$ \\
\hline AlSi11 0.5 wt\% oxide & 650 & 258.6 & 1.6 (ref. 28) & 25 & 40 \\
AlSi9 + 10 vol\% SiC & 700 & 190.2 & 2 (ref. 36) & 55 & 110 \\
AlSi9 + 20 vol\% SiC & 700 & 215.1 & 3.3 (ref. 36) & 42 & 139
\end{tabular}




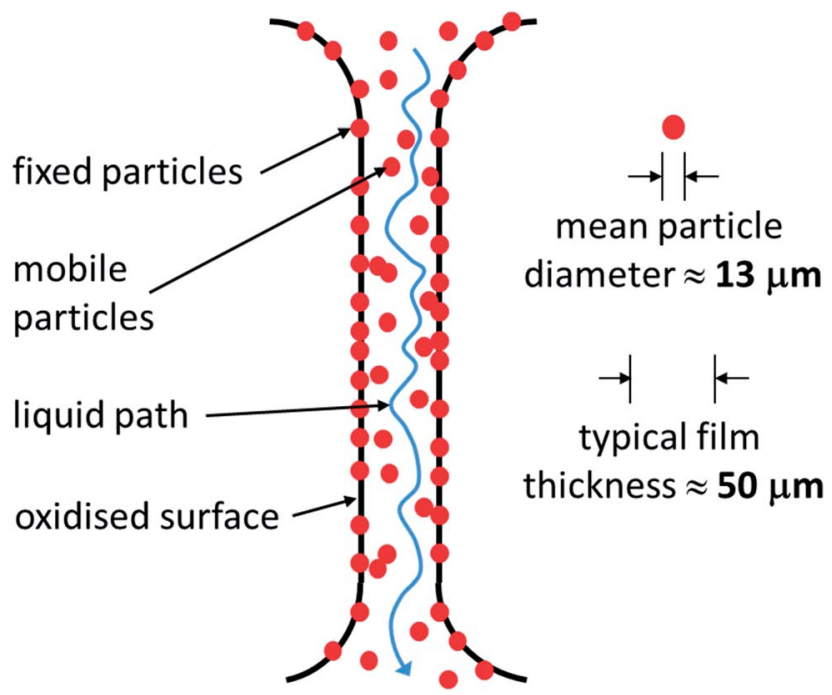

Fig. 4 Sketch of the structural effect in a foam film that increases the effective melt viscosity in a liquid metal film in a foam with floating and on the oxidised surface fixed SiC particles.

foamed AlSi $9+X \% \operatorname{SiC}(X=5,10$ and 20$)$ at $700{ }^{\circ} \mathrm{C}$ are also up to $38 \%$ lower than the corresponding bulk value without particles. ${ }^{31}$ There is also a small trend of increasing surface tension with increasing SiC concentration, but the trend is comparable to the standard deviations.

Like viscosity, the surface tension of aluminium is weakly affected by an addition of silicon. ${ }^{31}$ The present measurements show that further alloying and/or addition of particles reduces surface tension. Surface tensions of liquid AlSi11, AlSi6Cu4 and especially of AlSi9 + SiC foams are lower than those of the corresponding bulk alloys. The presence of partially wetting solid particles on the melt surfaces can indeed modify the values of tension. ${ }^{\mathbf{9 , 1 4}}$

A lower surface tension would support foam stability as the total interfacial energy is decreased and the driving force towards equilibrium (no foam) is slightly lower. However, since the effect is rather small compared to the increase of viscosity, surface tension is unlikely to play a dominant role in foam stabilisation.

\section{Conclusions}

- Microgravity experiments in which free drainage in a metal foam was characterised in situ by quantitative X-ray radioscopy after the onset of gravity yielded data that can be analysed in terms of the foam drainage equation.

- Values for the apparent surface tension and viscosity of liquid metal foams can be determined.

- The apparent viscosity of all melts in the foams is increased in comparison to corresponding bulk melts due to the structure of the metal films containing the melts.

- Addition of SiC particles also increases the apparent viscosity of the melts involved but this effect is one order of magnitude smaller than the structural effect.
- Oxides and SiC particles also reduce surface tension to a much smaller extent than viscosity.

- This methodology is also general in its approach, requiring only time-dependent liquid profiles and could be applied to other systems of interest such as polymeric foams.

\section{Acknowledgements}

We acknowledge Jörg Weise (Fraunhofer Bremen) for providing the thixocast AlSi6Cu4 precursors and Simon Cox for advice and assistance with drainage simulations. Funding by the European Space Agency (ESA) via microgravity application project ' $\mu$ gFOAM' (AO-99-075) and especially the support of Olivier Minster are gratefully acknowledged. Stefan Hutzler acknowledges support from COST Action MP1106 Smart and Green Interfaces.

\section{Notes and references}

1 A. Saint-Jalmes, S. Marze, M. Safouane, D. Langevin, S. J. Cox and D. Weaire, Microgravity Sci. Technol., 2006, 18, 108-111.

2 T. Wubben, J. Banhart and S. Odenbach, Microgravity Sci. Technol., 2002, 13, 36-42.

3 H. Caps, H. Decauwer, M. L. Chevalier, G. Soyez, M. Ausloos and N. Vandewalle, Eur. Phys. J. B, 2003, 33, 115-119.

4 B. M. Somosvári, P. Bárczy, J. Szóke, P. Szirovicza and T. Bárczy, Colloids Surf., A, 2011, 382, 58-63.

5 F. Garcia-Moreno, C. Jiménez, M. Mukherjee, P. Holm, J. Weise and J. Banhart, Colloids Surf., A, 2009, 344, 101-106.

6 F. Garcia-Moreno, M. Fromme and J. Banhart, Adv. Eng. Mater., 2004, 6, 416-420.

7 F. Garcia-Moreno, A. Rack, L. Helfen, T. Baumbach, S. Zabler, N. Babcsan, J. Banhart, T. Martin, C. Ponchut and M. Di Michiel, Appl. Phys. Lett., 2008, 92, 134104.

8 F. Garcia-Moreno, M. Mukherjee, C. Jiménez, A. Rack and J. Banhart, Metals, 2011, 2, 10-21.

9 F. Garcia-Moreno, E. Solorzano and J. Banhart, Soft Matter, 2011, 7, 9216-9223.

10 A. Stocco, F. Garcia-Moreno, I. Manke, J. Banhart and D. Langevin, Soft Matter, 2011, 7, 631-637.

11 J. Banhart and D. Weaire, Phys. Today, 2002, 55, 37-42.

12 V. Gergely and T. W. Clyne, Acta Mater., 2004, 52, 3047-3058.

13 C. Körner, M. Arnold and R. F. Singer, Mater. Sci. Eng., A, 2005, 396, 28-40.

14 A. Haibel, A. Rack and J. Banhart, Appl. Phys. Lett., 2006, 89, 154102.

15 G. Kaptay, Colloids Surf., A, 2004, 230, 67-80.

16 T. Wübben and S. Odenbach, Colloids Surf., A, 2005, 266, 207-213.

17 D. H. Kirkwood, Int. Mater. Rev., 1994, 39, 173-189.

18 J. P. Anson, R. A. L. Drew and J. E. Gruzleski, Metall. Mater. Trans. B, 1999, 30, 1027-1032.

19 C. Garcia-Cordovilla, E. Louis and A. Pamies, J. Mater. Sci., 1986, 21, 2787-2792.

20 G. Verbist, D. Weaire and A. M. Kraynik, J. Phys.: Condens. Matter, 1996, 8, 3715-3731. 
$21 \mathrm{~J}$. Weise, H. Stanzick and J. Banhart, presented in part at the Cellular Metals: Manufacture, Properties, Applications, Berlin, Germany, 2003.

22 J. Banhart and J. Baumeister, Production Methods for Metallic Foams, Proc. Materials-Research-Society Symposium on Porous and Cellular Materials for Structural Applications, San Francisco, 1998, 121-132.

23 V. Gergely and B. Clyne, Adv. Eng. Mater., 2000, 2, 175178.

24 F. James and M. Roos, Comput. Phys. Commun., 1975, 10, 343-367.

25 M. Haesche, J. Weise, F. Garcia-Moreno and J. Banhart, Mater. Sci. Eng., A, 2008, 480, 283-288.

26 A. M. Kraynik, D. A. Reinelt and F. van Swol, Phys. Rev. E: Stat., Nonlinear, Soft Matter Phys., 2003, 67, 031403.

27 D. Weaire and S. Hutzler, The Physics of Foams, Oxford University Press, 1999.

28 O. Brunke, A. Hamann, S. J. Cox and S. Odenbach, J. Phys.: Condens. Matter, 2005, 17, 6353-6362.

29 G. Bradley, PhD thesis, Trinity College Dublin, 2000.
30 C. J. Smithells, Smithells Metals Reference Book, Elsevier Butterworth-Heinemann, 2004.

31 J. Goicoechea, C. Garcia-Cordovilla, E. Louis and A. Pamies, J. Mater. Sci., 1992, 27, 5247-5252.

32 M. J. Assael, J. Phys. Chem. Ref. Data, 2006, 35, 285.

33 E. Gebhardt and K. Detering, Z. Metallkd., 1959, 50, 379-385.

34 W. R. D. Jones and W. L. Bartlett, J. Inst. Met., 1952, 81, 145-152.

35 K. K. Kalazhokov, Z. K. Kalazhokov and K. B. Khokonov, Tech. Phys., 2003, 48, 272-273.

36 M. C. Flemings, Metall. Trans. B, 1991, 22, 269-293.

37 E. Gebhardt, M. Becker and S. Dorner, Z. Metallkd., 1953, 44, 510-514.

38 R. F. Brooks, A. T. Dinsdale and P. N. Quested, Meas. Sci. Technol., 2005, 16, 354-362.

39 R. P. Chhabra and D. K. Sheth, Z. Metallkd., 1990, 81, 264-271. 40 E. N. d. C. Andrade, Philos. Mag., 1934, 17, 497-511.

41 A. Dudka, F. Garcia-Moreno, N. Wanderka and J. Banhart, Acta Mater., 2008, 56, 3990-4001.

42 S. Asavavisithchai and A. R. Kennedy, J. Colloid Interface Sci., 2006, 297, 715-723. 